\title{
The Simplest Double Slit: Interference and Entanglement in Double Photoionization of $\mathrm{H}_{2}$
}

\author{
D. Akoury ${ }^{1,2}$, K. Kreidi ${ }^{1}$, T. Jahnke ${ }^{1}$, Th. Weber ${ }^{1,2}$, A. Staudte ${ }^{1}$, \\ M. Schöffler ${ }^{1}$, N. Neumann ${ }^{1}$, J. Titze ${ }^{1}$, L. Ph. H. Schmidt ${ }^{1}$, A. Czasch ${ }^{1}$, \\ O. Jagutzki ${ }^{1}$, R.A. Costa Fraga ${ }^{1}$, R.E. Grisenti ${ }^{1}$, R. Díez Muiño ${ }^{3}$, \\ N.A. Cherepkov ${ }^{4}$, S.K. Semenov ${ }^{4}$, P. Ranitovic ${ }^{5}$, C.L. Cocke ${ }^{5}$, T. Osipov ${ }^{2}$, \\ H. Adaniya ${ }^{2}$, J.C. Thompson ${ }^{6}$, M.H. Prior ${ }^{2}$, A. Belkacem ${ }^{2}$, \\ A. L. Landers ${ }^{6}$, H. Schmidt- Böcking ${ }^{1}$, and R. Dörner ${ }^{1 *}$ \\ ${ }^{1}$ Institut für Kernphysik, University Frankfurt, \\ Max von Laue Str 1, D-60438 Frankfurt, Germany \\ ${ }^{2}$ Lawrence Berkeley National Lab., Berkeley CA 94720 \\ ${ }^{3}$ Centro de Fsica de Materiales and Donostia International \\ Physics Center, 20018 San Sebastián, Spain \\ ${ }^{4}$ State University of Aerospace Instrumentation, \\ 190000 St. Petersburg, Russia \\ ${ }^{5}$ Dept of Physics, Kansas State Univ, Cardwell Hall, \\ Manhattan KS 66506 \\ ${ }^{6}$ Department of Physics, Auburn University Auburn AL 36849 \\ *To whom correspondence should be addressed; \\ E-mail: doerner@atom.uni-frankfurt.de.
}

The wave nature of particles is rarely seen in nature. One reason is their very short de Broglie wavelengths in most situations. However, even with wavelengths close to the size of their surroundings, they couple to their environment, e.g. by gravity, Coulomb interaction, or thermal radiation. These cou- 
plings shift the phase of the waves, often in an uncontrolled way, hence yielding varying amounts of decoherence i.e. loss of phase integrity. Decoherence is thought to be a main cause of the transition from quantum to classical behavior. How much interaction is necessary and how big an environment is needed to induce the onset of classical behavior? Here we show that a photoelectron and two protons form a minimum particle/slit system, and that a minimum environment can be no more than a single additional electron. We observe interference "fringes" in the angular distribution of a single electron and the loss of fringe visibility caused by its Coulomb interaction with a second electron. While, at the same time, the correlated momenta of the entangled electron pair continue to exhibit quantum interference.

\section{Introduction}

One of the most powerful paradigms in the exploration of quantum mechanics is the double slit experiment. Thomas Young was the first to perform such an experiment, as early as 1801, with light. It took until the late 1950's [1], long after the experimental proof of the wave nature of particles, for a similar experiment to be carried out with electrons. Today such experiments have been demonstrated for particles as heavy as $C_{60}$ [2] and for bound electrons inside a highly excited atom [3]. All of these experiments were aimed at a demonstration of double slit self interference for a single particle fully isolated from the environment. If however this ideal laboratory situation is relaxed and the quantum particles are put in contact with the environment in a controlled way, the quantum interference may be diminished so that the particles start behaving in an increasingly classical way [4, 5, 6]. Recently Arndt and coworkers [7] have beautifully demonstrated this phenomenon by sending heated $C_{60}$ clusters through a double slit. The hot molecules couple via emission of thermal photons to the environment, and a loss of 
interference as a function of their temperature is observed. The emission of the photon alters the relative phase between different pathways of the particle towards the detector, an effect referred to as decoherence. Such decoherence of a quantum system can be caused by single or multiple interactions with an external system [6]. Limiting cases are one single hard interaction causing the decoherence by entanglement with the external system, and multiple weak couplings to external perturbers (i.e., a bath) at the other extreme. A gradual transition between these two extremes has been beautifully demonstrated for photon scattering [6].

Here we experimentally demonstrate that a system of two electrons is already sufficient to observe the transition from a quantum interference pattern to a classical particle-like intensity distribution for an individual electron. The quantum coherence is not destroyed however, but remains in the entangled two-electron system. By measuring the correlated momenta of both particles we reveal this interference pattern which is otherwise concealed in the two body wave function.

The idea of using a homonuclear molecule as the slit-scattering center of a photoelectron goes back to a paper published in 1966 by Cohen and Fano [8]. Due to the coherence in the initial molecular state, the absorption of one photon by the homonuclear molecule launches two coherent electron waves at each of the protons of the molecule (Fig. $1 \mathrm{a}, \mathrm{b}$ ). The interference pattern of these waves should be visible in the angular distribution of the electron with respect to the molecular axis. In K-shell ionization of heavy diatomics, such as $\mathrm{N}_{2}$ and $\mathrm{O}_{2}$ as discussed in this 1966 paper, interference is only visible if the symmetry (gerade or ungerade) of the molecule with a single $1 \mathrm{~s}$ hole is resolved $([9,10])$. For ground state $\mathrm{H}_{2}^{+}$and $\mathrm{H}_{2}$ molecules, only gerade orbitals are populated, and thus these systems constitute clean cases where slitlike behavior is expected [11]. The originally proposed experiment on $\mathrm{H}_{2}$ [11] has not been done however, because it requires knowledge of the direction of the molecular axis $[12,13]$. A signature of the interference effect has nonetheless been beautifully observed in the wavelength 
dependence of electrons emitted from a randomly oriented sample of $\mathrm{H}_{2}$ molecules by ion impact ionization $[14,15]$.

Here we extend the idea of Cohen and Fano from single photoionization to double photoionization to study the two body interference of an electron pair. This electron pair is emitted via absorption of a single circularly polarized photon from the $\mathrm{H}_{2}$ molecule (eq. 1).

$$
h \nu+H_{2} \rightarrow 2 p^{+}+2 e^{-}
$$

The two electrons are distinguishable by their energy, which allows us to the study the interference pattern as a function of the interaction strength or momentum exchanged between the two particles.

Single photons from beamlines 4.0 or 11.0 at the Advanced Light Source (ALS) at Lawrence Berkeley National Laboratory (LBNL) were used to photo-eject both electrons of $\mathrm{H}_{2}$ molecules. A supersonic $\mathrm{H}_{2}$ gas jet was crossed with the photon beam. For each ionized molecule the vector momenta of all fragment particles, both ions and both electrons, were determined in coincidence. The orientation of the $\mathrm{H}_{2}$ molecule, or molecular double slit, was measured for each fragmentation by detecting the emission direction of the two protons. Once the two electrons are ejected, the protons rapidly fly apart along the molecular axis, driven by their mutual Coulomb repulsion. A multiparticle imaging technique (Cold Target Recoil Ion Momentum Spectroscopy, COLTRIMS) $[16,17]$ was used to detect all particles. The ions and electrons created in the intersection volume of photon and gas beam were guided by weak electric $(50 \mathrm{~V} / \mathrm{cm})$ and magnetic fields (8 Gauss) towards two separate multichannel plate detectors with delayline readouts [18]. From the position of impact and the time-of-flight, the initial vector momentum of each particle can be determined. Only three particles (two protons and one electron) need to be detected. The momentum of the second electron, in the present case the more energetic of the two, is deduced through momentum conservation of the total system. The Coulomb explosion 
of the two protons at the equilibrium distance of $\mathrm{H}_{2}$ of 1.4 a.u. yields a kinetic energy of about $10 \mathrm{eV}$ per proton [19] and the total electronic binding energy of $\mathrm{H}_{2}$ is about $30 \mathrm{eV}$. The experiment has been performed at two different photon energies of $E_{\gamma}=240 \mathrm{eV}$ and $E_{\gamma}=160 \mathrm{eV}$, leaving about $190 \mathrm{eV}$ and $110 \mathrm{eV}$ of energy to be shared among the two electrons, respectively. At the high photon energies under consideration here, double photoionization of $\mathrm{H}_{2}$ leads in most cases to one fast electron and one slow electron [20].

Fig. 1d shows, for ionization by $240 \mathrm{eV}$ photons, the measured angular distribution for a highly energetic electron (called here 1) of energy $E_{1}$ of $185 \mathrm{eV}<E_{1}<190 \mathrm{eV}$. The second electron, unobserved here, acquires only an energy of $E_{2}<5 \mathrm{eV}$. The angular distribution is in the plane perpendicular to the photon propagation vector and the molecular axis is oriented horizontally in that plane. (The data plotted include events where electron 1, and the molecular axis, lie within 10 degrees of the ideal plane normal to the photon propagation direction)

The experimental data show a strong interference pattern which qualitatively resembles that induced by a double slit. For the optical double slit experiment, where the interference results from a superposition of two coherent spherical waves, the intensity distribution is given by eq. 2.

$$
I\left(\Phi_{e-m o l}\right)=C \cos \left(k_{e} R / 2 \cos \left(\Phi_{e-m o l}\right)\right)
$$

In our case, $R$ is the internuclear distance (1.4 a.u. for $\left.\mathrm{H}_{2}\right), \Phi_{e-m o l}$ is the angle of electron emission with respect to the internuclear axis [12], $k_{e}$ is the momentum of the electron and $\mathrm{C}$ is a proportionality constant. An electron energy of $190 \mathrm{eV}$ (as in Fig. 1) corresponds to $k_{e}=3.75 a . u$. (a.u. atomic units). The double slit prediction of eq. 2 is shown by the blue line in the Fig. 1e.

The deviations from the double slit prediction can be understood from the somewhat more elaborate theoretical treatment shown in Fig. 1e. By treating the electrons as spherical waves, 
the simple approximation in eq. 2 neglects the fact that the electrons are ejected by circularly polarized light, and further that they must escape from the two center Coulomb potential of the two nuclei. The helicity of the light leads to a slight clockwise rotation of the angular distribution as seen in the experiment and in the more elaborate calculations. The Coulomb interaction with the nuclei has two major effects. First, the wavelength of the electron in the vicinity of the protons is shorter than the asymptotic value. This property modifies, in particular, the emission probability along the molecular axis due to a phase shift in the nearfield [21]. Second, the original partial wave emerging from one of the nuclei is scattered at the neighboring nucleus, thereby launching another partial wave. The final diffraction pattern is therefore the superposition of four (or more) coherent contributions: the primary waves from the left and right nuclei, and the singly or multiply scattered waves created subsequently in the molecular potential. We performed two calculations to take the helicity of the photon as well as multiple scattering effects into account. The first (red line in Fig. 1e) was based on the Random Phase Approximation (RPA) [22]. The second (black line in fig 1e) entailed a multiple scattering calculation, wherein a spherical wave is launched at one proton [23]. This wave is then multiply scattered in the two center potential of two protons, which is terminated at a boundary. The direct and multiple scattered waves are then coherently added and symmetrized. Although conceptually very different, both calculations account for all the relevant physical features: the two center interference determining the position of minima and maxima, the molecular potential altering the relative height of the peaks, and the helicity of the ionizing photon inducing a rotation. The details of the molecular potential differ in the calculations. The RPA uses a Hartree Fock potential whereas the multiple scattering calculation assumes two bare protons.

The full calculations treat emission of a single electron. Therefore, their good agreement with the experimental data (Fig. 1d) obtained from double ionization might be surprising. It suggests that the additional emission of a slow electron does not significantly alter the wave of 
the fast particle. For the particular case that the electron pair consists of a fast and a very slow electron, the diffraction of a coherent electron pair can be treated by simply neglecting the slow electron.

This simple one particle picture completely fails in scenarios where lower primary and higher scattered electron energies result in stronger coupling between the electrons. Fig. 2a and $2 \mathrm{~b}$ show results for different energy partitions of the first and second electron following ionization by $160 \mathrm{eV}$ photons. Whereas for $E_{1} \approx 110 \mathrm{eV}$ and $E_{2}<1 \mathrm{eV}$ the interference is still visible (Fig. 2a), it completely disappears when $E_{1} \approx 95 \mathrm{eV}$ and $5 \mathrm{eV}<E_{2}<25 \mathrm{eV}$ (Fig. 2b). In the latter case the distribution approaches the isotropic result without two center interference. By comparing these data to corresponding theoretical estimates (Fig. 2c,d), we can now show that the observed loss of interference contrast is a result of decoherence and not of the changing electron wavelength.

Coulomb interaction between two quantum mechanical systems (electron 1 and 2 in our case) does not destroy phases. It entangles the wavefunctions of the two subsystems (see e.g. $[24,5])$. In our experiment we observe both electrons in coincidence. Therefore we can investigate this entangled two particle system to search for the origin of the apparent loss of coherence in a single particle subsystem. Fig. 3 shows the correlation in this two body system. The horizontal axis is the angle of the fast electron momentum with respect to the molecular axis (i.e. the angle which is plotted in all other figures). The vertical axis is the angle between the two electron 's momenta. It may be helpful to think of the horizontal axis as the scattering of electron 1 by the double slit and the vertical axis as the scattering angle between the electrons. Striking interference patterns emerge in this display of the two particle wavefunction. No vestige of these patterns remains, however, if the distribution is integrated over the vertical axis.

When subsets of the data with restricted angular ranges of $\Phi_{e-e}=+70 \pm 20^{\circ}$ (Fig 3b,c) and $\Phi_{e-e}=-70 \pm 20^{\circ}$ (Fig. 3d,e) are examined, then the interference pattern is resurrected. 
However, depending on the angle between the electrons in the selected subset of the data, the interference pattern is tilted to the right (Fig 3c) or left (Fig 3e). Without the restriction of this relative angle, the shifted minima and maxima cancel each other out, leading to the almost isotropic distribution of Fig. 2b.

The interference maxima are concentrated along two horizontal lines. These lines of highest intensity lie at a relative angle of about $100^{\circ}$ between the two electrons. This distribution is a well known $[20,25]$ indication of the mechanism whereby the absorption of a single photon by one electron can induce its ejection as well as that of the other electron, following their binary collision. The angles $\Phi_{e-e}=90^{\circ}$ and $\Phi_{e-e}=-90^{\circ}$ correspond to a kick of the second electron either to the left or to the right. This strong electron-electron Coulomb interaction mediates the double ionization and creates an entanglement between the two electrons. Electron collisions of this sort in bound systems have been demonstrated directly in pump probe experiments [26].

This situation is an intramolecular version of a scattering event downstream of a double slit $[27,6]$. When either photons [6] or particles [27] are scattered from a beam after passage through a double slit, the scattering induces a phase shift which then leads to a shift of the interference pattern. If the momentum transfer is not measured in coincidence [6], the fringe visibility is lost. In this experiment both electrons are initially delocalized inside the molecule in a completely coherent single quantum state. Before photoabsorption both electrons are confined in the hydrogen groundstate, which is symmetric with respect to its two atomic centers. Thus we observe not a scattering between classical localized particles but a coherent entanglement of the wave function of the two electrons.

It is instructive to think of the electronic two body system as split into its subsystems, and to consider one subsystem as the environment of the other. The strong Coulomb interaction entangles the two subsystems and leads to a position dependent modification of phase of the single particle wavefunction inside each of the two subsystems. The entanglement of the elec- 
trons in the pair is directly visible in their mutual angular distribution, and is further evidenced by the observation that selecting the momentum of one electron makes the interference pattern of its partner reappear. In the spirit of discussions dating from the early history of quantum mechanics, one particle can be considered an observer which carries partial information about the other particle and its path through the double slit. The amount of which-way information exchanged between the particles is limited by the observer particle's de Broglie wavelength [28]. The key difference between the situation depicted in Fig. 2a, which shows interference, and Fig. 2b, which shows no interference, is that the wavelength of the second, unobserved electron, is much shorter in the latter case.

Our experiment thus reveals that a very small number of particles suffices to induce the emergence of classical properties, such as the loss of coherence. A four-body system, such as fragmented molecular hydrogen, acts as a double slit in the sense that coherence is lost in a subsystem of entangled electrons. Such a fundamental system facilitates study of the influence of interelectronic Coulomb interaction on the coherence properties of a single electron. In solid-state-based quantum computing devices, such electron-electron interaction represents a key challenge. One delightful aspect of the finite system investigated here is that it is fully tractable theoretically today $[29,30,31,32]$.

\section{References and Notes}

[1] C. Jönnson, Zeitschrift für Physik 161, 454 (1961).

[2] M. Arndt, et al., Nature 401, 680 (1999).

[3] M. Noel, C. Stroud, Phys. Rev. Lett. 92, 1532011 (2004).

[4] E. Joos, H. Zeh, Zeitschrift für Physik B59, 223 (1985). 
[5] W. Zurek, Rev. Mod. Phys. 75, 715 (2003).

[6] D. Kokorowski, A. Cronin, T. Roberts, D. Pritchard, Phys. Rev. Lett. 86, 2191 (2001).

[7] L. Hackermüller, K. Hornberger, B. Brezger, A. Zeilinger, M. Arndt, Nature 427, 711 (2004).

[8] H. Cohen, U. Fano, Phys. Rev. 150, 30 (1966).

[9] D. Rolles, et al., Nature 7059, 711 (2005).

[10] X. Liu, et al., J. Phys. B39, 4801 (2006).

[11] I. Kaplan, A. Markin, Sov. Phys. Dokl. 14, 36 (1969).

[12] M. Walter, J. Briggs, J. Phys. B32, 2487 (1999).

[13] J. Fernandez, O. Fojon, A. Palacios, F. Martn, Phys. Rev. Lett. 98, 043005 (2007).

[14] N. Stolterfoht, et al., Phys. Rev. Lett. 87, 0232011 (2001).

[15] D. Misra, et al., Phys. Rev. Lett. 92, 1532011 (2004).

[16] J. Ullrich, et al., Rep. Prog. Phys. 66, 1463 (2003).

[17] R. Dörner, et al., Physics Reports 330, 96 (2000).

[18] O. Jagutzki, et al., Nucl. Instr. Meth. A477, 244 (2002).

[19] T. Weber, et al., Nature 431, 437 (2004).

[20] A. Knapp, et al., Phys. Rev. Lett. 89, 0330041 (2002).

[21] G. Yudin, S. Chelkowski, A. Bandrauk, J. Phys B39, L17 (2006). 
[22] S. Semenov, N.A.Cherepkov, J. Phys. B36, 1409 (2003).

[23] R. D. M. no, D. Rolles, F. J. G. de Abajo, C. S. Fadley, M. A. V. Hove, J. Phys. B35, L359 (2002).

[24] T. Opatrny, G. Kurizki, Phys. Rev. Lett. 86, 3180 (2001).

[25] M. Pont, R. Shakeshaft, Phys. Rev. A51, R2676 (1995).

[26] S. Pisharody, R. Jones, Science 303, 813 (2004).

[27] K. Hornberger, et al., Phys. Rev. Lett. 90, 1604011 (2003).

[28] W. Wootters, W. Zurek, Phys. Rev. D19, 473 (1979).

[29] W. Vanroose, F. Martín, T. Rescigno, C. McCurdy, Science 310, 1787 (2005).

[30] F. Martin, et al., Science 315, 629 (2007).

[31] J. Colgan, M. Pindzola, F. Robicheaux, J. Phys. B37, L377 (2004).

[32] D. Dundas, J. Phys. B37, 2883 (2004).

[33] We are indebted to Michael Walter, John Briggs, Anatoli Kheifets, Uwe Becker, Daniel Rolles, Erich Joos, Kiyoshi Ueda, Markus Arndt, Markus Aspelmeyer for enlightening discussions. we acknowledge outstanding support by the staff of the Advanced Lights Source in particular by Elke Arenholz, Tony Young, Hendrik Bluhm and Tolek Tyliszczak. the work was supported by the Deutsche Forschungsgemeinschaft and by the Office of Basic Energy Sciences, Division of Chemical Sciences of the US DOE under contract DE-AC02-057CH11231. 


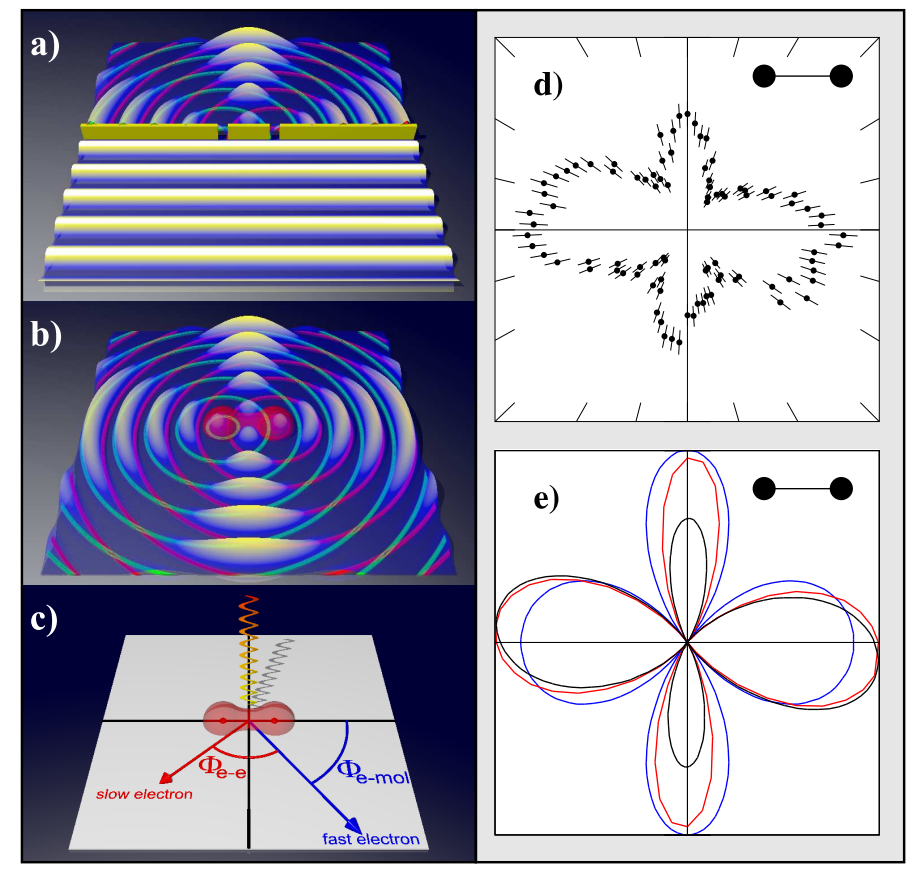

Figure 1: a) Schematic view of a double slit arrangement. A plane wave approaches the slit from the front. The slit separation is 1.4 a.u. (the internuclear distance in $\mathrm{H}_{2}$ ), and the wavelength is 3.75 a.u which corresponds to an electron energy of $190 \mathrm{eV}$. b) Photoionization by circularly polarized light launches a coherent spherical photoelectron wave at each nucleus of the molecule; the light propagates into the plane, c) Geometry of the present experiment; circularly polarized light comes from the top. All angular distributions shown in this paper are in the plane perpendicular to the photon propagation vector, $\Phi_{e-m o l}$ is the angle of the fast electron's trajectory to the molecular axis, and $\Phi_{e-e}$ is the angle between both electron trajectories. d) Measured electron angular distribution $\left(\Phi_{e-m o l}\right)$ of the faster electron from double photoionization of $\mathrm{H}_{2}$ by circularly polarized light. The orientation of the molecule is horizontal. Light propagates into the plane of the figure, the molecule is fixed $\pm 10^{\circ}$ within the plane shown, $E_{\gamma}=240 \mathrm{eV}$ and the energy of the slow electron $E_{2}=0 t o 5 \mathrm{eV}$, resulting in $E_{1}=185 t o 190 \mathrm{eV}$. e) Angular probability distributions derived from eq. 2 (blue line), RPA calculation (red), and multiple scattering calculation (black). 


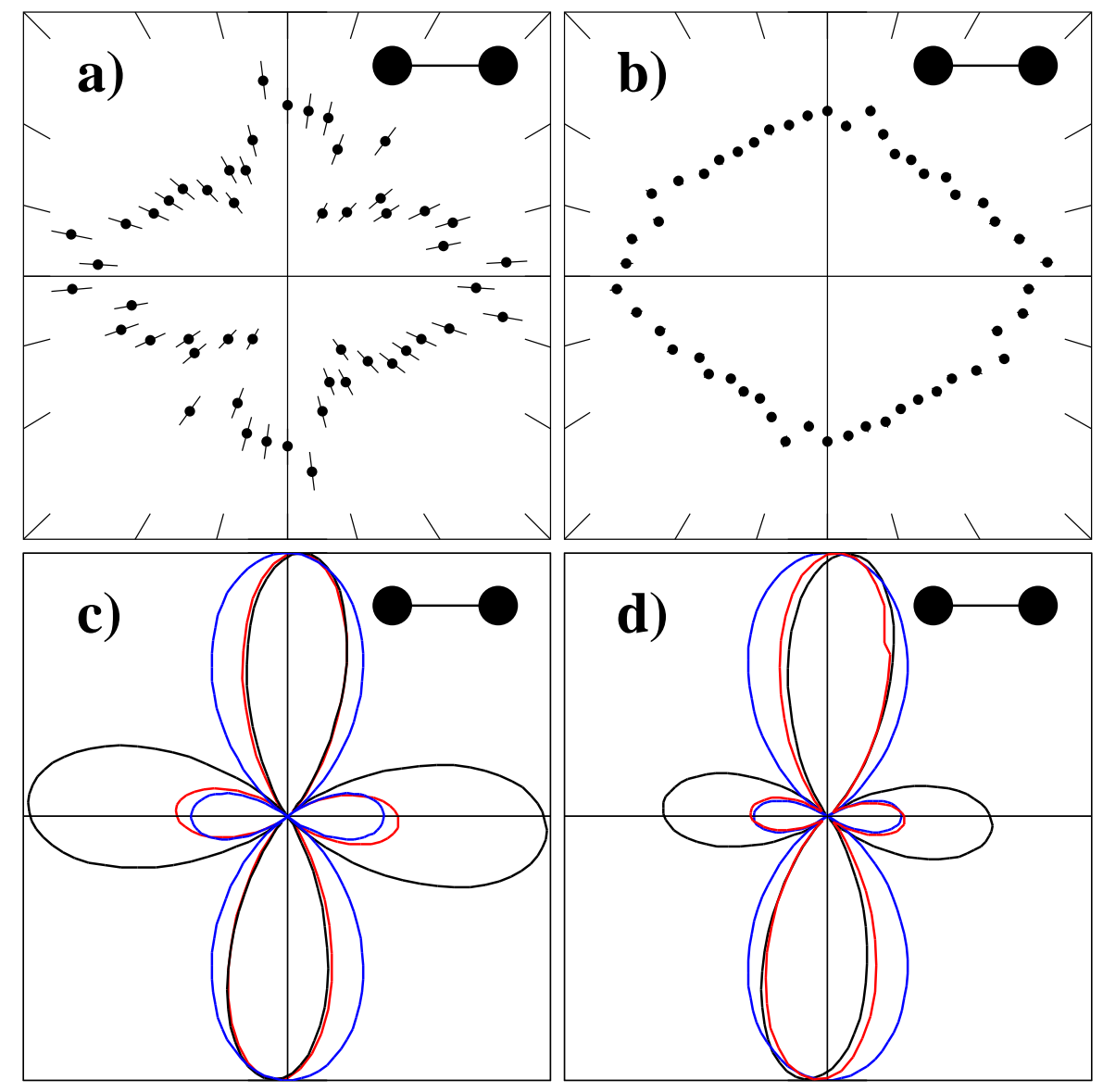

Figure 2: a) Electron angular distribution as in Fig. 1d but for $E_{\gamma}=160 \mathrm{eV}, E_{2}<1 \mathrm{eV}$ and $E_{1} \approx 110 \mathrm{eV}$, b) $E_{\gamma}=160 \mathrm{eV}, 5 \mathrm{eV}<E_{2}<25 \mathrm{eV}$ resulting in $E_{1} \approx 85-105 \mathrm{eV}$. c) and d) Angular distribution of a single electron of energy $E_{e}$ for the energy distributions in panels (a) and (b) respectively; eq. 2 (red), RPA calculation (blue), multiple scattering calculation (black). 


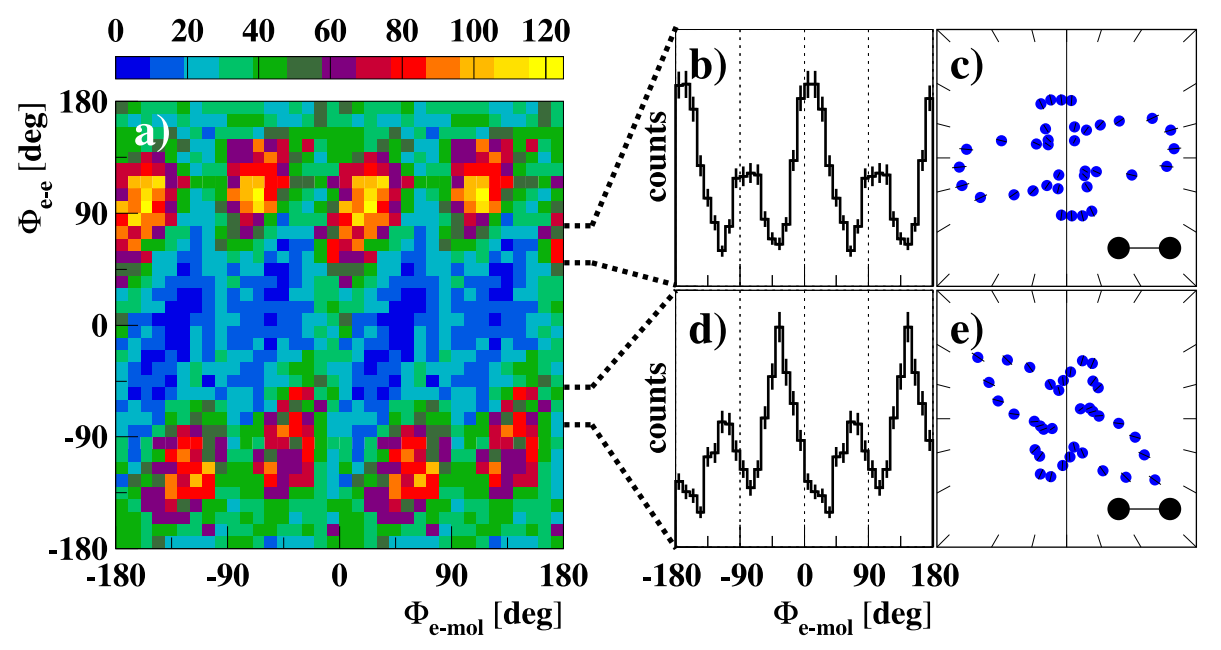

Figure 3: Correlation between both electrons for double photoionization of $\mathrm{H}_{2}$ at $E_{\gamma}=160 \mathrm{eV}$, $5 \mathrm{eV}<E_{2}<25 \mathrm{eV}$ corresponding to $E_{1} \approx 85-105 \mathrm{eV}$. a) horizontal axis: angle of fast electron to the molecular axis $\left(\Phi_{e 1 \mathrm{~mol}}\right)$ (see fig. 1c), vertical axis: angle $\Phi_{e-e}$ between the two electrons; both electrons and the molecule are selected to lie within $\pm 30^{\circ}$ of the polarization plane. Thus Fig. $2 \mathrm{~b}$ is a projection of this figure onto the horizontal axis. b) A projection of (a) onto the horizontal axis for $50^{\circ}<\Phi_{e-e}<80^{\circ}$. d) The analogous projection for $-80^{\circ}<\Phi_{e-e}<-50^{\circ}$. c) Polar presentation of the data shown in (b). e) Polar presentation of data shown in (d) 